\title{
Challenges of Combination Therapies in Alzheimer's Disease
}

Created by: Md. Sahab Uddin

Version received: 14 May 2020

\begin{abstract}
Alzheimer's disease (AD) is the leading cause of dementia worldwide. Until now, available therapeutic agents for $A D$ treatment only provide symptomatic treatment. Since $A D$ pathogenesis is multifactorial, use of a multimodal therapeutic intervention addressing several molecular targets of AD-related pathological processes seems to be the most practical approach to modify the course of AD progression. It has been demonstrated through numerous studies, that the clinical efficacy of combination therapy (CT) is higher than that of monotherapy. It is indeed difficult to combine several pharmacophores into a single molecule. It is essential to carry out long-duration randomized controlled trials to establish whether CT delays disease progression in early AD stages. Other factors also need to be assessed in CT, such as its potential neuroprotective effects, cost-effectiveness, and a more exhaustive estimation of its potential benefits on the patients at the end-stage of AD.
\end{abstract}

Alzheimer's disease (AD) is a complex neurodegenerative disorder, thus treating AD patients still remains a challenge. Presently, the approved AD treatments are limited to memantine and cholinesterase inhibitors (ChEls) or the combination of these two agents. Most of the newly tested drugs failed in phase III trials due to their incapacity to meet the target efficacy, even though they exhibited promising results in their initial studies. The complex nature of $A D$ is considered as one of the main reasons for the high failure rate of these new treatments ${ }^{[1]}$. Additionally, another reason for this high failure rate is our incomplete understanding of the numerous mechanisms involved in AD development and following neurodegeneration and the potential lack of efficacy of the available drugs. Since the combination of ChEls and memantine have encountered less success in case of AD treatment, thus targeting multiple pathways to treat AD has better chances of success. Therefore, additional studies examining rational combinations of agents ought to be continued.

As add-ons to standard-of-care therapy, multiple current studies are currently exploring the effects of symptomatic agents or disease-modifying therapies. Nonetheless, a combination of two or more agents targeting different mechanisms and providing synergistic effects has better chances to treat AD. However, only a limited number of these agents are under clinical trials [2] .

The use of ChEl-memantine combination therapy (CT) in patients with mild-to-moderate AD has not been demonstrated conclusively. Randomized controlled trials (RCTs) exhibited similar short-term performances for $\mathrm{CT}$ and monotherapy ${ }^{[\underline{3}]}$, while long-term observational analyses support the effectiveness of the $\mathrm{CT}$ to decrease the rate of cognitive decline and the level of dependence and denote that $\mathrm{CT}$ is more effective when started early and maintained for a long period [4][ㅁ][]][]]. Thus, longduration RCTs are needed to confirm whether a CT applied in the early stages of AD can delay the progression of this disease. The potential long-term benefits of a CT should be more apparent after two years when the rate of deterioration is more evident $[\underline{8}$.

CT might also diminish the doses used of the individual drugs with an eventual decrease in the costs and the side effects of the treatments. The design of adaptive and innovative clinical trials might be interesting for the potential development of therapeutic combinations over the disease progression, using one set of agents for preclinical $A D$, another for early-stage $A D$, and yet another for $A D$ dementia. Collectively, challenges in AD treatment have guided the current therapeutic approaches toward the evaluation of new drugs as an add-on to standard-of-care and the repurpose of currently approved therapeutic agents which are indicated for other therapeutic conditions as well as the combination of agents that target different pathways $[\underline{[}]$. 
Even though most of the add-on trials with potential disease-modifying drugs have failed so far, results in this area indicate that success may be further obtained, predominantly in case of neurotrophic agents and anti-amyloid strategies. Cerebrolysin, anti-A $\beta$ vaccines and insulin may constitute beneficial agents which require additional studies in the future. Combinations of anti-amyloid and/or anti-tau interventions with neurotrophic agents have not yet been investigated but can lead to additive or synergistic effects. Further studies with drugs in prodromal or early AD stages may require long follow-up periods to prove their efficacy as the rates of progression of the disease and the clinical deterioration of patients are highly variable and rather slow $[\underline{2}]$.

\section{References}

1. Sahab Uddin; Tanvir Kabir; Devesh Tewari; Bijo Mathew; Lotfi Aleya; Emerging signal regulating potential of small molecule biflavonoids to combat neuropathological insults of Alzheimer's disease. Science of The Total Environment 2020, 700, 1-11, 10.1016/j.scitotenv.2019.134836.

2. X Antón Alvarez; Carlos Linares; Eliezer Masliah; Combination Drug Therapy for the Treatment of Alzheimer's Disease. European Neurological Review 2012, 7, 92-101, 10.17925/enr.2012.07.02.92.

3. Anton P. Porsteinsson; George T Grossberg; Jacobo Mintzer; Jason T Olin; Memantine treatment in patients with mild to moderate Alzheimer's disease already receiving a cholinesterase inhibitor: a randomized, double-blind, placebocontrolled trial. Current Alzheimer Research 2008, 5, 83-89, 10.2174/156720508783884576.

4. Alireza Atri; Lynn W. Shaughnessy; Joseph J. Locascio; John H. Growdon; Long-term Course and Effectiveness of Combination Therapy in Alzheimer Disease. Alzheimer Disease \& Associated Disorders 2008, 22, 209-221, 10.1097/wad.0b013e31816653bc.

5. Oscar L. Lopez; J T Becker; Abdus Wahed; J Saxton; R A Sweet; D A Wolk; William Klunk; Steven T. DeKosky; Longterm effects of the concomitant use of memantine with cholinesterase inhibition in Alzheimer disease. Journal of Neurology, Neurosurgery \& Psychiatry 2009, 80, 600-607, 10.1136/jnnp.2008.158964.

6. David Wilkinson; A review of the effects of memantine on clinical progression in Alzheimer's disease. International Journal of Geriatric Psychiatry 2011, 27, 769-776, 10.1002/gps.2788.

7. Susan Rountree; Wenyaw Chan; Valory Pavlik; Eveleen Darby; Samina Siddiqui; Rachelle S. Doody; Persistent treatment with cholinesterase inhibitors and/or memantine slows clinical progression of Alzheimer disease. Alzheimer's Research \& Therapy 2009, 1, 7, 10.1186/alzrt7.

8. Sophie Gillette; Sandrine Andrieu; Fati Nourhashemi; Virginie Gardette; Nicola Coley; Christelle Cantet; Serge Gauthier; Pierre Jean Ousset; Bruno Vellas; REAL.FR study group; et al. Long-term progression of Alzheimer's disease in patients under antidementia drugs. Alzheimer's \& Dementia 2011, 7, 579-592, 10.1016/j.jalz.2011.02.009.

9. Jeffrey L. Cummings; Gary Tong; Clive Ballard; Treatment Combinations for Alzheimer's Disease: Current and Future Pharmacotherapy Options. Journal of Alzheimer's Disease 2019, 67, 779-794, 10.3233/jad-180766.

\section{Keywords}

Alzheimer's disease; combination therapy; memantine; cholinesterase inhibitors

(c) 2020 by the author(s). Distribute under a Creative Commans CC BY license 